\title{
Protective effect of polyphenols in an inflammatory process associated with experimental pulmonary fibrosis in mice
}

\author{
Daniela Impellizzeri ${ }^{1}$, Elena Talero ${ }^{2}$, Rosalba Siracusa ${ }^{1}$, Antonio Alcaide ${ }^{2}$, Marika Cordaro ${ }^{1}$, \\ Jose Maria Zubelia ${ }^{3}$, Giuseppe Bruschetta ${ }^{1}$, Rosalia Crupi $^{1}$, Emanuela Esposito ${ }^{1}$, Salvatore Cuzzocrea ${ }^{1}$ * \\ and Virginia Motilva ${ }^{2}$ \\ ${ }^{1}$ Department of Biological and Environmental Sciences, University of Messina, 98166 Messina, Italy \\ ${ }^{2}$ Department of Pharmacology, Faculty of Pharmacy, University of Seville, 41012 Seville, Spain \\ ${ }^{3}$ PoliNat Inc., 35259 Ingenio, Las Palmas, Canary Islands, Spain
}

(Submitted 2 March 2015 - Final revision received 26 May 2015 - Accepted 16 June 2015 - First published online 12 August 2015)

\section{Abstract}

Polyphenols have been described to have a wide range of biological activities, and many reports, published during recent years, have highlighted the beneficial effects of phenolic compounds, illustrating their promising role as therapeutic tools in several acute and chronic disorders. The purpose of study was to evaluate, in an already-assessed model of lung injury caused by bleomycin (BLM) administration, the role of resveratrol and quercetin, as well as to explore the potential beneficial properties of a mango leaf extract, rich in mangiferin, and a grape leaf extract, rich in dihydroquercetin (DHQ), on the same model. Mice were subjected to intra-tracheal administration of BLM, and polyphenols were administered by oral route immediately after BLM instillation and daily for $7 \mathrm{~d}$. Treatment with resveratrol, mangiferin, quercetin and DHQ inhibited oedema formation and body weight loss, as well as ameliorated polymorphonuclear infiltration into the lung tissue and reduced the number of inflammatory cells in bronchoalveolar lavage fluid. Moreover, polyphenols suppressed inducible nitric oxide synthase expression, and prevented oxidative and nitroxidative lung injury, as shown by the reduced nitrotyrosine and poly (ADP-ribose) polymerase levels. The degree of apoptosis, as evaluated by Bid and Bcl-2 balance, was also suppressed after polyphenol treatment. Finally, these natural products down-regulated cyclo-oxygenase-2, extracellular signal-regulated kinase phosphorylated expression and reduced NF- $\kappa \mathrm{Bp} 65$ translocation. Our findings confirmed the anti-inflammatory effects of resveratrol and quercetin in BLM-induced lung damage, and highlight, for the first time, the protective properties of exogenous administration of mangiferin and DHQ on experimental pulmonary fibrosis.

\section{Key words: Polyphenols: Oxidative stress: Inflammation: Lung injury: Apoptosis}

Idiopathic pulmonary fibrosis (IPF) is the most common among interstitial pneumonias of unknown origin and is one of the most aggressive interstitial lung diseases (ILD). A number of exogenously administered agents including commonly used drugs are known to induce an iatrogenic form of pulmonary fibrosis $^{(1)}$. Bleomycin (BLM) is an anti-tumour agent currently used in humans for the treatment of Hodgkin's lymphoma. Repeated administration of this drug may lead to lung inflammation and fibrosis as side-effects; for this reason, it is used to reproduce a lung fibrosis state. In fact, this model is characterised by an early predominantly neutrophilic inflammatory response, increased fibroblast proliferation and enhanced collagen deposition because of increased collagen synthesis and decreased collagen degradation ${ }^{(2)}$. In addition, it has been shown that fibroproliferative activity co-exists with inflammation, and that the major proliferative phase occurs during the 1st week after BLM-induced injury ${ }^{(3)}$. BLM is able to alter the balance between the oxidant and the antioxidant defence system in the lung. In this model, hydroxyl radicals, superoxide anion radical, hydrogen peroxide and peroxynitrite are increased by BLM administration ${ }^{(4)}$. Reactive oxygen species (ROS) over-production causes the peroxidation of membrane lipids and DNA damage ${ }^{(5)}$, which ultimately results in tissue injury, with the activation of several intra-cellular signalling pathways leading to the production of proinflammatory cytokines $^{(6)}$. Moreover, NO synthase (iNOS), which is the main source of NO during inflammation, and nitrotyrosine, a by-product of peroxynitrite activity, are upregulated in IPF lungs ${ }^{(7)}$. Polyphenols are a large structural class of natural compounds, ubiquitously distributed in plant sources,

Abbreviations: BAL, bronchoalveolar lavage; BLMbleomycin; COX-2, cyclo-oxygenase-2; DHQ, dihydroquercetin; iNOS, inducible NO synthase; IPF, idiopathic pulmonary fibrosis; MPO, myeloperoxidase; PARP, poly (ADP-ribose) polymerase; pERK, phosphorylated extracellular signal-regulated kinase; ROS, reactive oxygen species.

* Corresponding author: Professor S. Cuzzocrea, email salvator@unime.it 
and have long been important components of the human diet. They are the most promising natural compounds to combat metabolic syndromes including age-related inflammatory states $^{(8)}$. Many polyphenols are efficient antioxidants and antiinflammatory molecules by virtue of their ability to directly scavenge inflammation-derived radicals, to increase the expression of antioxidants and to block the production of inflammatory cytokine by modulating the activities of specific transcription factors ${ }^{(9)}$. Resveratrol (trans-3,5,4'-trihydroxystilbene) is a phytoalexin polyphenol produced naturally by several plants and is available in various dietary food stuffs such as grapes, berries, red wine and nuts. It is characterised by multiple health-promoting properties including antioxidative, anti-cancer, anti-inflammatory, anti-ageing, blood sugarlowering and beneficial cardiovascular effects ${ }^{(10-12)}$. Quercetin $\left(3,3^{\prime}, 4^{\prime}, 5,7\right.$-pentahydroxyflavone) is a prototypical polyphenolic plant flavonoid found in onions, broccoli, tomatoes, apples and berries. It possesses a wide variety of protective properties (e.g. antioxidant, anti-inflammatory, anti-allergic and anti-proliferative $)^{(13)}$. Mangiferin (1,3,6,7-tetrahydroxyxanthone $\mathrm{C} 2-\beta$-D-glucoside) is a natural glucosyl xanthone commonly found in various parts of Mangifera indica and is also known to exhibit numerous pharmacological activities including hepatoprotective, anti-carcinogenic, anti-diabetic, anti-viral, antioxidant, anti-apoptotic and anti-inflammatory properties ${ }^{(14,15)}$. Dihydroquercetin (DHQ) ((2R,3R)-2-(3,4-dihydroxyphenyl)3,5,7-trihydroxy-2,3-dihydrochromen-4-one) is a flavonoid commonly found in onions ${ }^{(16)}$, milk, thistle ${ }^{(17)}$ and French maritime bark $^{(18)}$. This polyphenol is classified as an antioxidant ${ }^{(19)}$ and meets two of the three criteria for effective radical scavenging ability - the presence of the 0 -dihydroxy structure in the B ring conferring stability and the 5- and 7-OH groups with 4-oxo function in the $\mathrm{A}$ and $\mathrm{C}$ rings that are responsible for a maximum radical scavenging potential. In addition to its antioxidative effect, DHQ has been shown to have beneficial properties in cancer, CVD and liver diseases ${ }^{(20)}$. Previous studies have reported the effects of resveratrol and quercetin in experimental models of pulmonary fibrosis. In this line, resveratrol administered either as a preventive or as a therapeutic strategy - attenuated oxidative injury and fibrosis due to $\mathrm{BLM}^{(21,22)}$. Likewise, treatment with quercetin, or liposomal quercetin, which exhibits improved water solubility and selective accumulation in lung tissues ${ }^{(23)}$, prevented lung injury in the same model ${ }^{(24,25)}$. The beneficial activities of these polyphenols have been attributed to the inhibition of leucocyte infiltration, oxidative stress, pro-inflammatory cytokine levels - such as TNF- $\alpha$, IL- $1 \beta$ and IL- $6-$ as well as the reduction of hydroxyproline content, considered as an important indicator to directly reflect collagen content in pulmonary fibrosis. Recently, mangiferin has been reported to have inhibitory effects on airway inflammation in a model of allergic asthma as well as in a sepsis-induced lung injury model ${ }^{(26,27)}$. However, no studies have dealt with the effects of mangiferin, or DHQ, on BLM-induced lung injury. Thus, the purpose of the present study was to further evaluate, in an already-assessed model of lung injury caused by BLM administration, the role of resveratrol and quercetin as well as to explore the potential beneficial properties of a mango leaf extract, rich in mangiferin, and a grape leaf extract, rich in DHQ, on the same model.

\section{Methods}

\section{Animals}

Male CD-1 (CD1(ICR)) mice (25-35 g; Harlan Nossan) were housed in a controlled environment and were provided with standard rodent chow and water. Mice were housed in stainless-steel cages in a room maintained at $22 \pm 1^{\circ} \mathrm{C}$ with a $12 \mathrm{~h}$ light $-12 \mathrm{~h}$ dark cycle. The animals were acclimatised to their environment for 1 week and had ad libitum access to tap water and rodent standard diet (Harlan Nossan). All efforts were made to minimise animal suffering and to use only the number of animals necessary to produce reliable scientific data.

\section{Ethical statement}

This study was approved by the University of Messina Review Board for the care of animals and was carried out in accordance with the Declaration of Helsinki. Procedures involving animals and their care were conducted in conformity with the Italian regulations on protection of animals used for experimental and other scientific purpose (D.M. 116192) as well as with the EEC regulations (O.J. of E.C. L 358/1 12/18/1986).

\section{Experimental groups}

Mice were randomised into six experimental groups.

(i) Bleomycin + vehicle group ( $n$ 10). Mice were subjected to BLM-induced lung injury as described below and received saline orally $(0.9 \%, \mathrm{w} / \mathrm{v})$, immediately after BLM instillation and every day starting from day 1 until day 7 .

(ii) Resveratrol group ( $n$ 10). Same as the BLM+vehicle group, but resveratrol was administered by oral route $(50 \mathrm{mg} / \mathrm{kg})^{(28)}$, immediately after BLM instillation and daily for $7 \mathrm{~d}$.

(iii) Mangiferin group ( $n$ 10). Same as the BLM+vehicle group but Mangifera indica leaf extract, containing $\geq 60 \%$ mangiferin, was administered by oral route $(10 \mathrm{mg} / \mathrm{kg})^{(27)}$, immediately after BLM instillation and daily for $7 \mathrm{~d}$.

(iv) Quercetin group ( $n$ 10). Same as the BLM + vehicle group, but quercetin was administered by oral route $(10 \mathrm{mg} /$ $\mathrm{kg})^{(29)}$, immediately after BLM instillation and daily for $7 \mathrm{~d}$.

(iv) Dihydroquercetin group ( $n$ 10). Same as the BLM+vehicle group but Vitis vinifera leaf extract, containing $\geq 90 \%$ total dihydroquercetins - was administered by oral route $(10 \mathrm{mg} / \mathrm{kg})$, immediately after BLM instillation and daily for $7 \mathrm{~d}$

(vi) Sham + vehicle group ( $n$ 10). Identical to the BLM + vehicle group, but animals received intra-tracheal administration of saline $(0.9 \%, \mathrm{w} / \mathrm{v})$, instead of BLM, and were treated with vehicle orally after saline administration and daily for $7 \mathrm{~d}$.

\section{Materials}

All the extracts were kindly donated by PoliNat SL. In brief, standardised extracts were obtained by hydro-alcoholic extraction of plant materials, followed by concentration, spray drying and sieving. Bioactive content was quantified (w/w\%) by 
UPLC-DAD. All the manufacturing procedures were performed under GMP compliance.

Unless otherwise stated, all the other compounds were obtained from Sigma-Aldrich Company. All the chemicals were of the highest commercial grade available. All stock solutions were prepared in non-pyrogenic saline $(0.9 \% \mathrm{NaCl}$; Baxter Healthcare Ltd) or $10 \%$ ethanol (Sigma-Aldrich).

\section{Induction of lung injury by bleomycin}

Mice received a single intra-tracheal instillation of saline (0.9\%, $\mathrm{w} / \mathrm{v}$ ) or saline containing BLM sulphate ( $1 \mathrm{mg} / \mathrm{kg}$ body weight) at end-expiration in a volume of $100 \mu \mathrm{l}$, and the liquid administration was followed immediately by $300 \mu \mathrm{l}$ of air to ensure delivery to the distal airways; drug administration started on day 1 and animals were killed using pentobarbitone overdose on day 7 after BLM administration.

\section{Bronchoalveolar lavage}

On day 7 after BLM or saline solution administration, mice were euthanised, terminal plasma samples were collected and the tracheas were immediately cannulated with an I.V. polyethylene catheter (Neo Delta Ven 2. delta Med; Neo Delta) equipped with a twenty-four-gauge needle on a 1-ml syringe. Lungs were lavaged once with $0.5 \mathrm{ml}$ D-PBS (GIBCO). In $>95 \%$ of the mice, the recovery volumes were over $0.4 \mathrm{ml}$. The bronchoalveolar lavage (BAL) fluid was centrifuged at $800 \mathrm{rpm}$, the supernatants were stored and the pelleted cells were collected. Total BAL cells were enumerated by counting on a haemocytometer in the presence of trypan blue. Cytospins were prepared from re-suspended BAL cells. Cytospins of BAL cells were made by centrifuging 50000 cells onto microscope slides using a Shandon Cytospin 3 (Shandon). The slides were allowed to air dry and were then stained with Diff-Quick Stain Set (Diff-Quick; Baxter Scientific). A total of 400 cells were counted from randomly chosen high-power microscope fields for each sample.

\section{Measurement of lung oedema}

The wet lung weights were measured $7 \mathrm{~d}$ after injection of BLM by careful excision of the lung from other adjacent extraneous tissues. The lungs were exposed for $48 \mathrm{~h}$ at $180^{\circ} \mathrm{C}$ and the dry weight was subsequently measured. Water content of the lungs was subsequently calculated as the ratio of wet:dry weight of the tissue.

\section{Histological examination}

Lung biopsies were taken $7 \mathrm{~d}$ after injection of BLM. After fixation at room temperature in buffered formaldehyde solution (10\% in PBS), the sections were prepared and stained with haematoxylin-eosin and observed using an Axiovision Zeiss (Zeiss) microscope. The grades of lung fibrosis were scored on a scale from 0 to 8 by examining randomly chosen sections, with five fields per sample at a magnification of $100 \times$. Criteria for grading lung fibrosis were as follows ${ }^{(30)}$ : grade 0 , normal lung; grade 1, minimal fibrous thickening of alveolar or bronchiolar walls; grade 3, moderate thickening of the walls without obvious damage to lung architecture; grade 5, increased fibrosis with definite damage to lung structure and formation of fibrous bands or small fibrous masses; grade 7 , severe distortion of lung structure and large fibrous areas; grade 8 , total fibrous obliteration of fields.

\section{Myeloperoxidase assay}

Myeloperoxidase (MPO) activity was determined $7 \mathrm{~d}$ after injection of BLM. The lung was removed, weighed, homogenised in a solution containing $0.5 \%$ hexadecyl trimethyl ammonium bromide dissolved in $10 \mathrm{~mm}$-potassium phosphate buffer ( $\mathrm{pH} 7$ ) and centrifuged for $30 \mathrm{~min}$ at $20000 \boldsymbol{g}$ at $4^{\circ} \mathrm{C}$. An aliquot of the supernatant was then allowed to react with a solution of tetramethylbenzidine $(1.6 \mathrm{~mm})$ and $0.1 \mathrm{~mm}-\mathrm{H}_{2} \mathrm{O}_{2}$. The rate of change in absorbance was measured spectrophotometrically at $650 \mathrm{~nm}$. MPO activity was defined as the quantity of enzyme degrading $1 \mu \mathrm{mol}$ of peroxide $/ \mathrm{min}$ at $37^{\circ} \mathrm{C}$ and was expressed in units per gram weight of wet tissue ${ }^{(31)}$.

Immunohistochemical evaluation of nitrotyrosine and poly (ADP-ribose) polymerase

Tyrosine nitration, an index of the nitrosylation of proteins by peroxynitrite and/or ROS, was determined immunohistochemically as previously described ${ }^{(20)}$. At the end of the experiment, the tissues were fixed in $10 \%(\mathrm{w} / \mathrm{v})$ PBS-buffered formaldehyde, and $8-\mu \mathrm{m}$ sections were prepared from paraffinembedded tissues. After de-paraffinisation, endogenous peroxidase was quenched with $0.3 \%(\mathrm{v} / \mathrm{v})$ hydrogen peroxide in $60 \%(\mathrm{v} / \mathrm{v})$ methanol for $30 \mathrm{~min}$. The sections were permeabilised with $0.1 \%(\mathrm{w} / \mathrm{v})$ Triton X-100 in PBS for $20 \mathrm{~min}$. Non-specific adsorption was minimised by incubating the sections in $2 \%(\mathrm{v} / \mathrm{v})$ normal goat serum in PBS for $20 \mathrm{~min}$. Endogenous biotin- or avidin-binding sites were blocked by sequential incubation for $15 \mathrm{~min}$ with biotin and avidin (DBA), respectively. Sections were incubated overnight with antinitrotyrosine polyclonal antibody (1:500 in PBS (v/v)) and anti-poly (ADP-ribose) polymerase (PARP) goat polyclonal antibody (1:500 in PBS (v/v)). Sections were washed in PBS and incubated with secondary antibody. Specific labelling was detected using biotin-conjugated goat anti-rabbit IgG and avidin-biotin peroxidase complex (DBA). In order to confirm that the immunoreactions for nitrotyrosine were specific, some sections were also incubated with the primary antibody (anti-nitrotyrosine) in the presence of excess nitrotyrosine (10 mm). In order to verify the binding specificity for PARP, some sections were also incubated with the primary antibody alone (no secondary) or with the secondary antibody alone (no primary). In these situations, no positive staining was found in the sections, indicating that the immunoreactions were positive in all the experiments carried out.

Western blots for Bid, BCl-2, inducible nitric oxide synthase, cyclo-oxygenase-2, phosphorylated extracellular signal-regulated kinase, NF- KBp 65 and $/ \kappa B-\alpha$

In brief, lung tissues from each mouse were suspended in extraction buffer A containing 0.2 mм-PMSF, $0 \cdot 15 \mu \mathrm{m}$ pepstatin A, 
$20 \mu \mathrm{m}$ leupeptin and $1 \mu \mathrm{m}$ sodium orthovanadate, homogenised at the highest setting for $2 \mathrm{~min}$, and centrifuged at $1000 \boldsymbol{g}$ for $10 \mathrm{~min}$ at $4^{\circ} \mathrm{C}$. Supernatants represented the cytosolic fraction. The pellets, containing enriched nuclei, were re-suspended in buffer $\mathrm{B}$ containing $1 \%$ Triton $\mathrm{X}-100$, 150 mм-NaCl, 10 mм-Tris-HCl, pH 7.4, 1 mм-EGTA, 1 mм-EDTA, $0.2 \mathrm{~mm}$-PMSF, $20 \mu \mathrm{m}$ leupeptin and $0.2 \mathrm{~mm}$-sodium orthovanadate. After centrifugation at $15000 \mathrm{~g}$ for $30 \mathrm{~min}$ at $4{ }^{\circ} \mathrm{C}$, the supernatants containing the nuclear protein were stored at $-80^{\circ}$ $\mathrm{C}$ for further analysis. The levels of NF- $\kappa \mathrm{Bp} 65$ were quantified in the nuclear fraction from the lung tissues. The levels of Bid, Bcl-2, iNOS, COX-2, I $\kappa \mathrm{B}-\alpha$ and phosphorylated extracellular signal-regulated kinase (pERK) were quantified in the cytosolic fraction from the lung tissues. The filters were blocked with $1 \times \mathrm{PBS}, 5 \%(\mathrm{w} / \mathrm{v})$ non-fat dried milk for $40 \mathrm{~min}$ at room temperature, and they were subsequently probed with specific antibodies anti-Bid (1:500; Santa Cruz Biotechnology), anti-Bcl-2 (1:500; Santa Cruz Biotechnology), anti-I $\kappa$ B- $\alpha$ (1:500; Santa Cruz Biotechnology), anti-NF- $\kappa$ Bp65 (1:500; Santa Cruz Biotechnology), anti-iNOS (1:500; Santa Cruz Biotechnology), anti-COX-2 (1:500; Santa Cruz Biotechnology) and anti-pERK (1:500; Santa Cruz Biotechnology) in $1 \times$ PBS, $5 \%$ (w/v) non-fat dried milk and $0 \cdot 1 \%$ Tween 20 at $4^{\circ} \mathrm{C}$ overnight. Membranes were incubated with peroxidase-conjugated bovine anti-mouse IgG secondary antibody or peroxidase-conjugated goat anti-rabbit IgG (1:2000; Jackson ImmunoResearch Laboratories) for $1 \mathrm{~h}$ at room temperature. To ascertain that blots were loaded with equal amounts of protein lysates, they were also incubated in the presence of the antibody against $\beta$-actin (1:2000; SigmaAldrich). The relative expression of the bands of Bid (approximately $23 \mathrm{kDa}$ ), Bcl-2 (approximately $29 \mathrm{kDa}$ ), iNOS (approximately $130 \mathrm{kDa}$ ), COX-2 (approximately $70 \mathrm{kDa}$ ), pERK (approximately $46 \mathrm{kDa}$ ), NF- $\kappa \mathrm{Bp} 65$ (approximately $65 \mathrm{kDa}$ ) and $\mathrm{I} \kappa \mathrm{B}-\alpha$ (approximately $37 \mathrm{kDa}$ ) were quantified by densitometric scanning of the X-ray films with a GS-700 imaging densitometer (GS-700; Bio-Rad) and a computer programme (Molecular Analyst; IBM).

\section{Statistical analysis}

All the values in the figures and text are expressed as mean values with their standard errors of $n$ observations, where $n$ represents the number of animals studied. In the experiments involving histology, the figures shown are representative of at least three sections (histological colouration) of the tissues collected from all the animals in each group. Data sets were examined by one-way ANOVA, and group means were then compared with that of the BLM control (Bleo) using Dunnett's test. A $P$ value of $<0.05$ was considered statistically significant.

\section{Results}

Effects of resveratrol, mangiferin, dihydroquercetin and quercetin on bleomycin-induced lung injury, fluid content and body weight

After $7 \mathrm{~d}$ of BLM injection, the pulmonary lesions observed in mice consisted of multifocal areas of severe inflammation and intense fibrosis, characterised by massive neutrophilic infiltration and plasma cells extending through the lung epithelium (Fig. 1(b)), when compared with lung sections taken from sham animals (Fig. 1(a)). In contrast, treatment with resveratrol, mangiferin, DHQ and quercetin revealed a lesssevere pattern of pulmonary lesions, consisting of moderate inflammation and slight fibrosis (Fig. 1(c)-(f)). The histological scoring of fibrosis severity in the lung samples showed in BLMchallenged mice a severe degree of injury when compared with sham-operated mice. Polyphenol administration significantly reduced the histopathological score (Fig. 1(g)).

BLM administration caused an increase in wet:dry lung weight ratio, due to infiltration of inflammatory cells and oedema, compared with sham-operated mice. On the contrary, treatment with resveratrol, mangiferin, DHQ and quercetin showed a significant decrease in wet:dry lung weight ratio (Fig. 1(h)). The severe lung injury caused by BLM administration was also associated with a significant loss in body weight, whereas treatment with polyphenols attenuated body weight loss (Fig. 1(i)).

\section{Effects of resveratrol, mangiferin, dihydroquercetin and quercetin on total and differential cell counts in the bronchoalveolar lavage}

To further assess the intensity of inflammatory responses in the lung, we examined the amount of leucocytes and differential cells in the BAL fluid. After $7 \mathrm{~d}$ of BLM administration, mice showed a significant increase in the total number of inflammatory cells (leucocyte account) in the BAL (compared with the sham group) (Fig. 2(a)), which was reduced after treatment with resveratrol, mangiferin, DHQ and quercetin. In addition, a marked increase in the differential cell counts was evident in BLM-induced animals. In contrast, all polyphenol-treated mice that underwent BLM tracheal instillation showed a decreased content of BAL cellularity when evaluated by differential cell count on cytospin preparations (Fig. 2(b)-(e)).

\section{Effects of resveratrol, mangiferin, dihydroquercetin and quercetin on myeloperoxidase activity}

The lung fibrosis caused by BLM administration was also characterised by an increase in MPO activity, an indicator of neutrophil accumulation in the lung (Fig. 2(f)). Resveratrol at $50 \mathrm{mg} / \mathrm{kg}$, mangiferin and DHQ at $10 \mathrm{mg} / \mathrm{kg}$ significantly reduced the degree of polymorphonuclear leucocytes infiltration (determined as increase in MPO activity, U/g wet tissue) in the inflamed lung (Fig. 2(f)). However, treatment with quercetin did not significantly affect neutrophil infiltration in inflamed lungs.

\section{Effects of resveratrol, mangiferin, dihydroquercetin and quercetin on nitrotyrosine and poly (ADP-ribose) polymerase localisation}

Immunohistochemical analysis of lung sections obtained from mice treated with BLM revealed positive staining for nitrotyrosine, localised mainly in the nuclei of inflammatory cells 
(a)

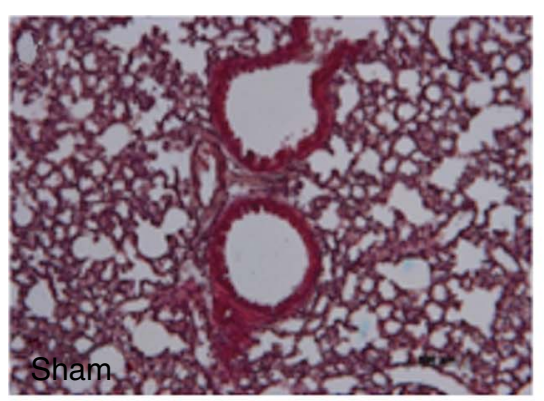

(d)

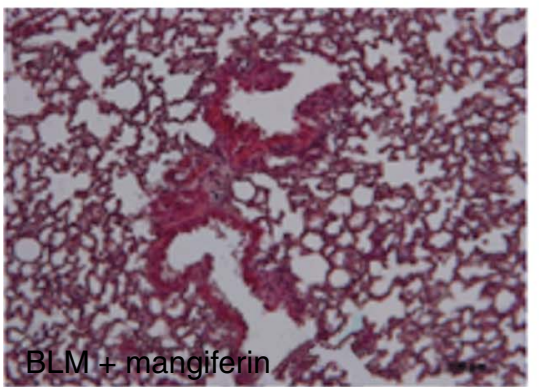

(b)

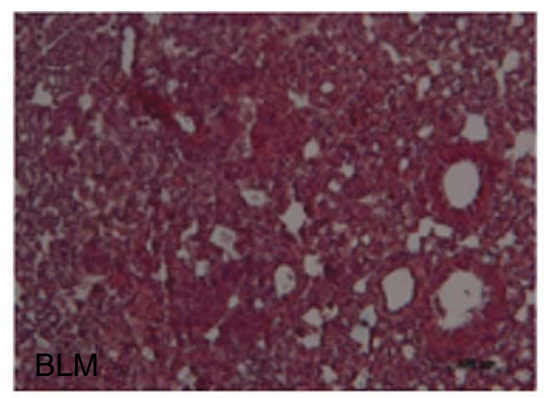

(e)

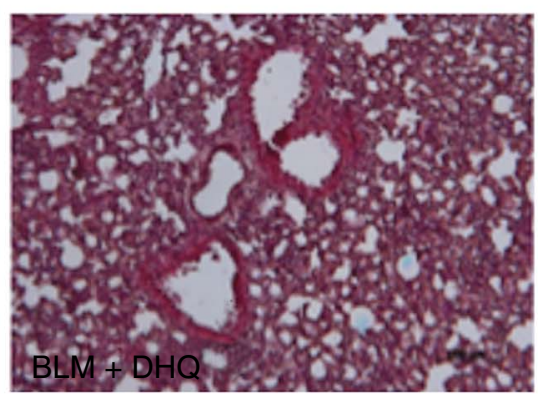

(c)

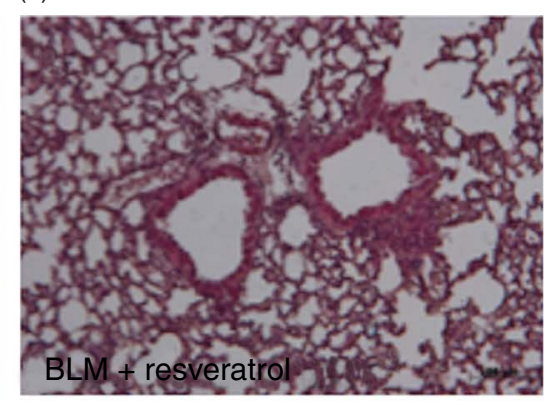

(f)

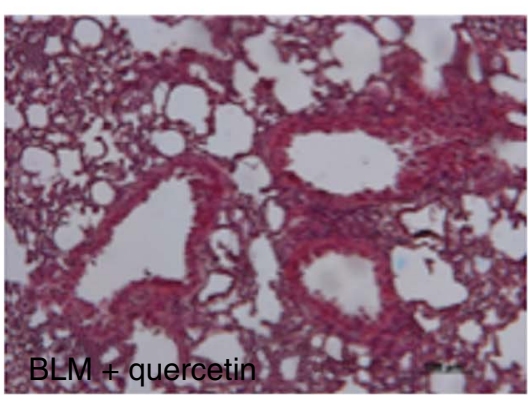

(g)

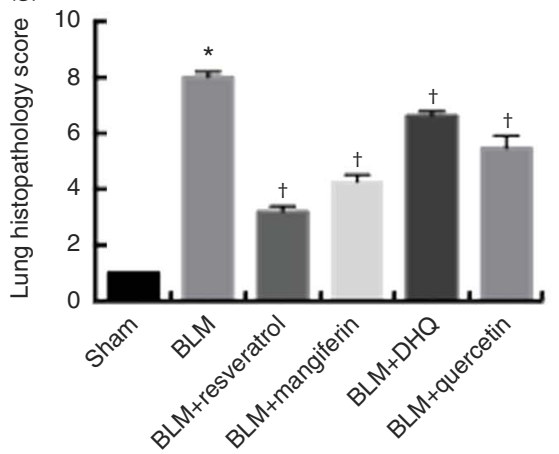

(h)

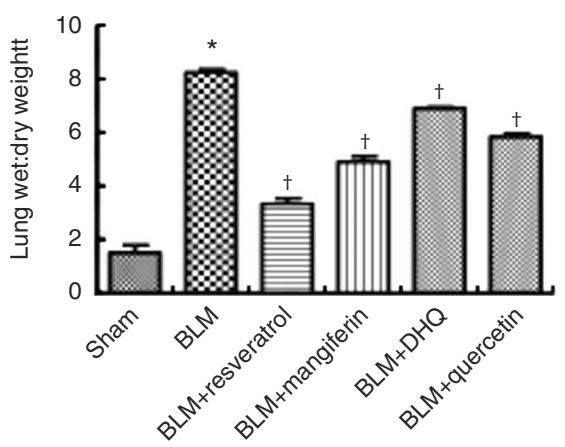

(i)

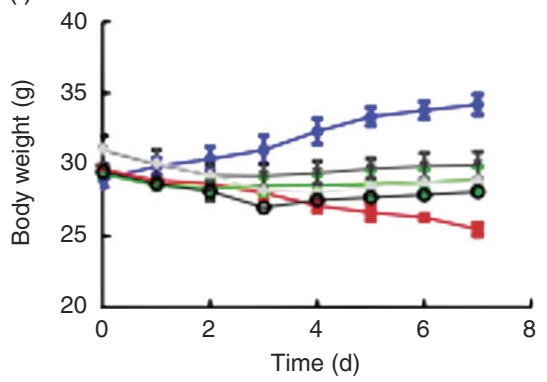

Fig. 1. Effects of polyphenols on bleomycin (BLM)-induced lung injury, fluid content and body weight. Haematoxylin-eosin staining of lung sections from BLM-instilled mice revealed significant tissue damage ((b) and see histological score (g)) when compared with sham-operated animals ((a) and see histological score (g)). Resveratrol, mangiferin, dihydroquercetin (DHQ) and quercetin administration caused a decrease in pulmonary lesions, consisting of moderate inflammation and slight fibrosis ((c-f) and see histological score (g)). BLM administration also caused an increase in wet:dry lung weight ratio in relation to sham-operated mice. On the contrary, treatment with resveratrol, mangiferin, $\mathrm{DHQ}$ and quercetin showed a significant decrease of this parameter (h). Moreover, the severe lung injury caused by BLM administration was associated with a significant loss in body weight, whereas resveratrol, mangiferin, DHQ and quercetin treatment attenuated body weight loss (i). Data are expressed as mean values and standard deviations from $n 10$ mice for each group. ${ }^{*} P<0.05 v$. sham; $† P<0.05 v$. BLM. $\longrightarrow-$, Sham; $\multimap, \mathrm{BLM} ; \multimap, \mathrm{BLM}+$ resveratrol; $\multimap, \mathrm{BLM}+$ mangiferin; —, BLM + DHQ; —O-, BLM + quercetin.

(Fig. 3(b)). In contrast, positive staining for nitrotyrosine was significantly reduced in the lungs from BLM-treated mice that had been treated with resveratrol, mangiferin, DHQ and quercetin (Fig. 3(c)-(f), see densitometry analysis in Fig. 3(g)). Immunohistochemical analysis of lung sections obtained from mice treated with BLM also revealed positive staining for PARP (Fig. 4(b)). However, reduced staining for PARP was found in the lungs from BLM-treated mice that had been treated with the different polyphenols (Fig. 4(c)-(f), see densitometry analysis in Fig. $4(\mathrm{~g})$ ). There was no staining for either nitrotyrosine or PARP in the lungs from the sham group (Fig. 3(a) and 4(a), respectively).
Effects of resveratrol, mangiferin, dihydroquercetin and quercetin on apoptosis in bleomycin-treated mice

To test whether BLM damage was associated with cell death, we evaluated the appearance of the pro-apoptotic protein Bid and the anti-apoptotic protein Bcl-2 by Western blot analysis. Bid levels were appreciably increased in the lung tissue collected from mice subjected to BLM injection (Fig. 5(a)). In contrast, treatment with resveratrol and mangiferin significantly prevented BLM-induced Bid expression (Fig. 5(a)). No changes in this protein levels were observed in mice subjected to DHQ and quercetin, as shown in Fig. 5(a). Expression of the 
(a)

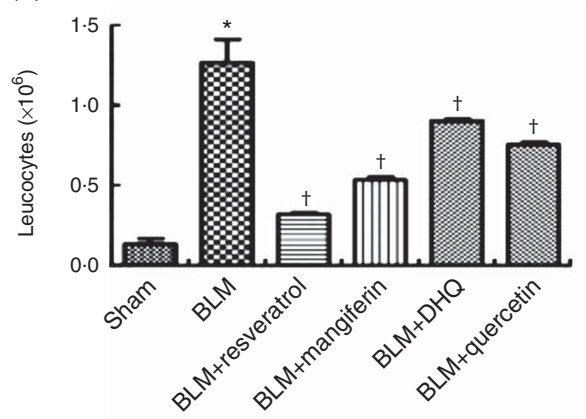

(d)

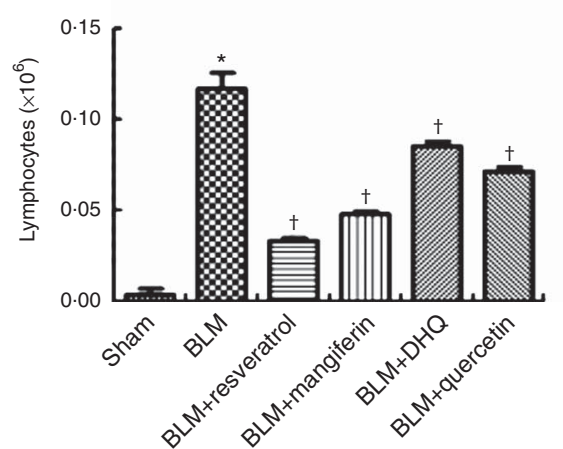

(b)

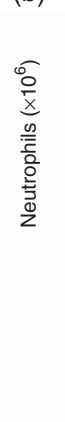

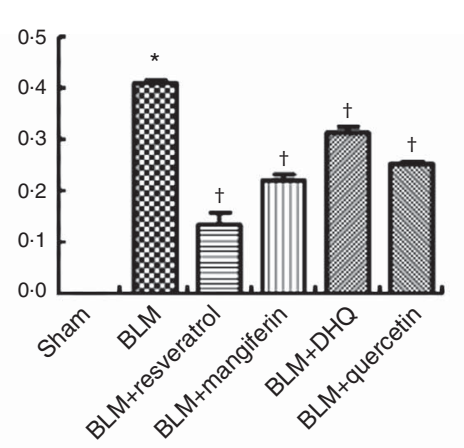

(c)

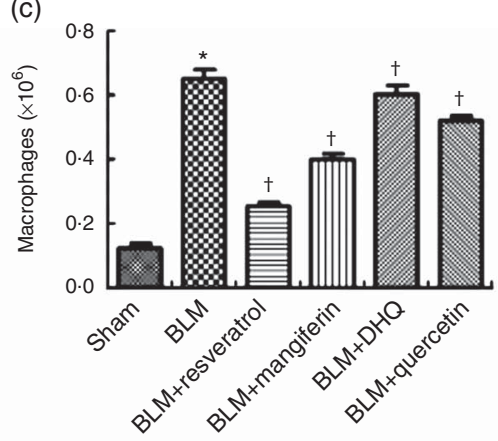

(e)

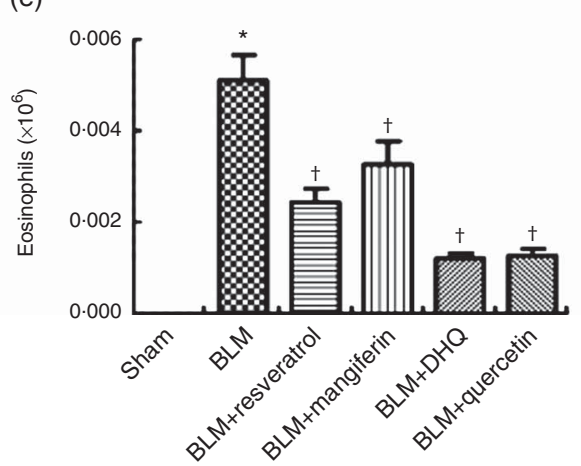

(f)

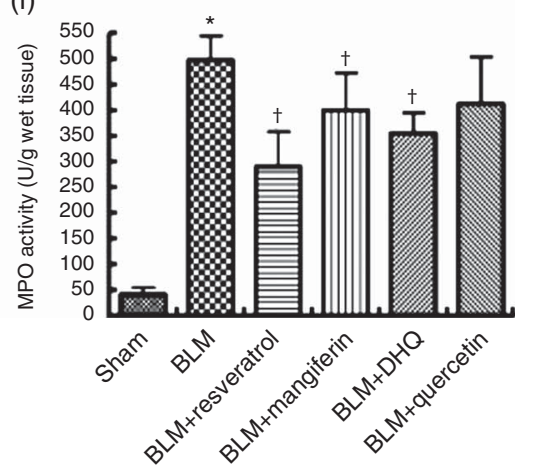

Fig. 2. Effects of polyphenols on total and differential cellularity of bronchoalveolar lavage (BAL) and myeloperoxidase (MPO) activity. Total BAL cellularity for sham, bleomycin (BLM)-administered mice and polyphenol-treated mice is shown in (a). Differential cells counts are also shown for neutrophils (b), macrophages (c), lymphocytes (d) and eosinophils (e) per millilitre of BAL fluid. In addition, MPO activity (f) was increased by BLM administration when compared with lung tissues obtained from sham animals. In contrast, tissue sections from BLM-administered mice and treated with resveratrol, mangiferin and dihydroquercetin (DHQ) showed a decreased MPO activity. Data are expressed as mean values and standard deviations from $n 10$ mice for each group. ${ }^{*} P<0.05 v$. sham; $† P<0.05 v$. BLM.

anti-apoptotic protein Bcl-2 was significantly diminished in BLM-treated mice compared with the sham group. However, polyphenol administration induced a marked increase in Bcl-2 expression (Fig. 5(b)).

\section{Effects of resveratrol, mangiferin, dihydroquercetin and quercetin on nitric oxide synthase expression in bleomycin-treated mice}

To determine the levels of NO produced during lung injury, iNOS expression was evaluated by Western blotting in the lung sections $7 \mathrm{~d}$ after BLM injection. We observed an increased expression of iNOS in the group subject to BLM compared with the control group (Fig. 6(a)), whereas treatment with resveratrol, mangiferin, DHQ and quercetin significantly reduced this enzyme expression (Fig. 6(a)).

\section{Expression levels of cyclo-oxygenase-2 after treatment with resveratrol, mangiferin, dihydroquercetin and quercetin}

To study the involvement of the inflammatory process following BLM injury, we examined the ability of resveratrol, mangiferin, DHQ and quercetin to influence injury-induced cyclooxygenase-2 (COX-2) expression. Western blot analysis showed that BLM injection significantly increased the expression of COX-2; however, the levels of this protein were markedly attenuated in the groups of mice treated with resveratrol, mangiferin and DHQ (Fig. 6(b)). In contrast, quercetin administration was not able to reduce COX-2 expression (Fig. 6(b)).

\section{Expression levels of phosphorylated extracellular signal-regulated kinase after treatment with resveratrol, mangiferin, dihydroquercetin and quercetin}

To investigate the cellular mechanisms by which treatments with resveratrol, mangiferin, DHQ and quercetin may attenuate the development of lung injury, we also evaluated the activation of MAPK through phosphorylation of ERK by Western blot. Quantitative analysis of this protein showed that BLM injection significantly increased the expression of pERK; however, the protein levels were markedly attenuated in the groups of mice treated with resveratrol, mangiferin, DHQ and quercetin (Fig. 6(c)).

\section{Expression levels of $N F-\kappa B$ and $/ \kappa B-\alpha$ after treatment with resveratrol, mangiferin, dihydroquercetin and quercetin}

We evaluated $\mathrm{I} \kappa \mathrm{B}-\alpha$ degradation and NF- $\kappa \mathrm{Bp} 65$ translocation by Western blot analysis to investigate the cellular mechanisms whereby treatment with resveratrol, mangiferin, DHQ and 
(a)

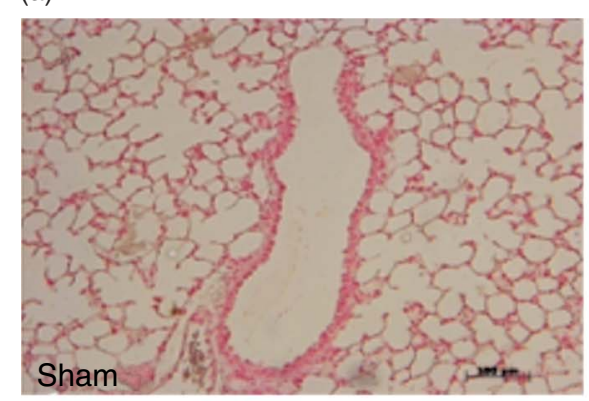

(d)

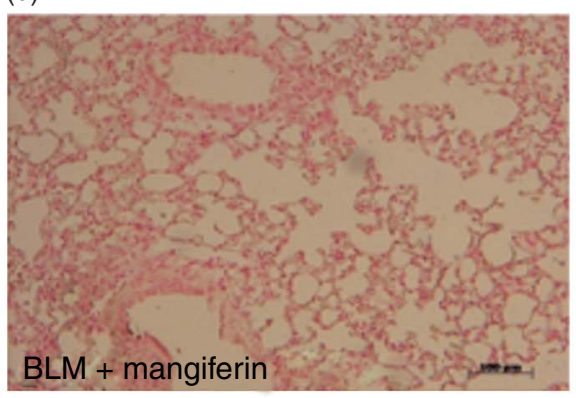

(b)

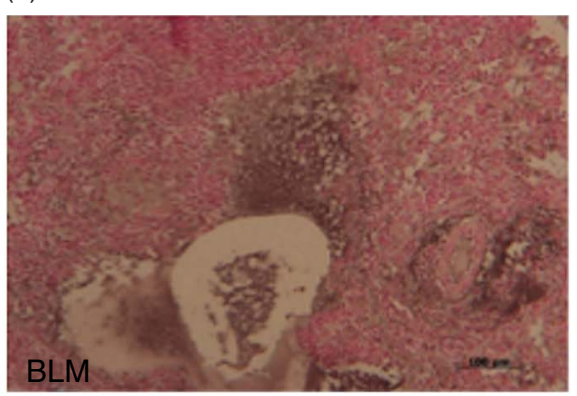

(e)

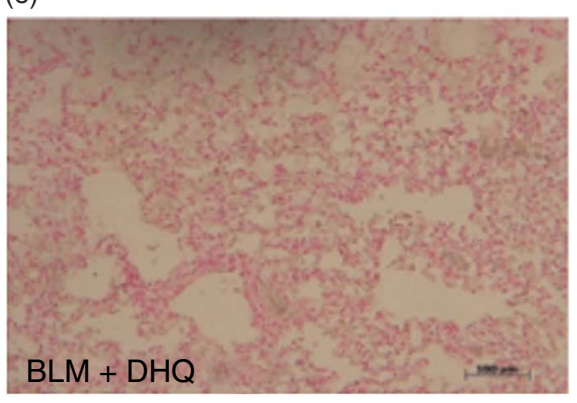

(c)

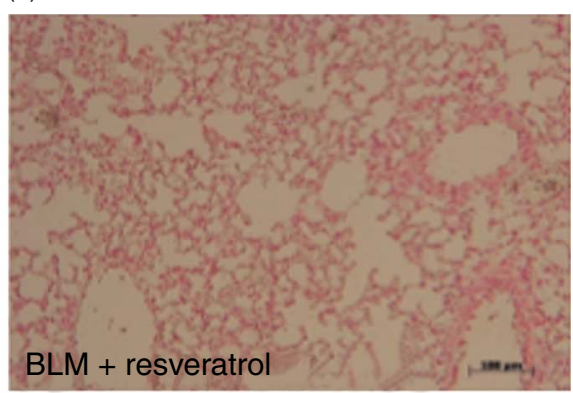

(f)

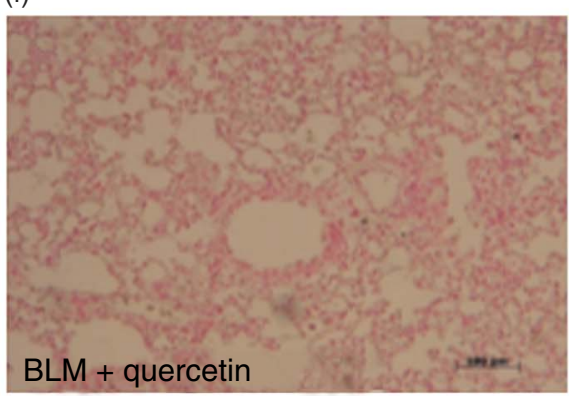

(g)

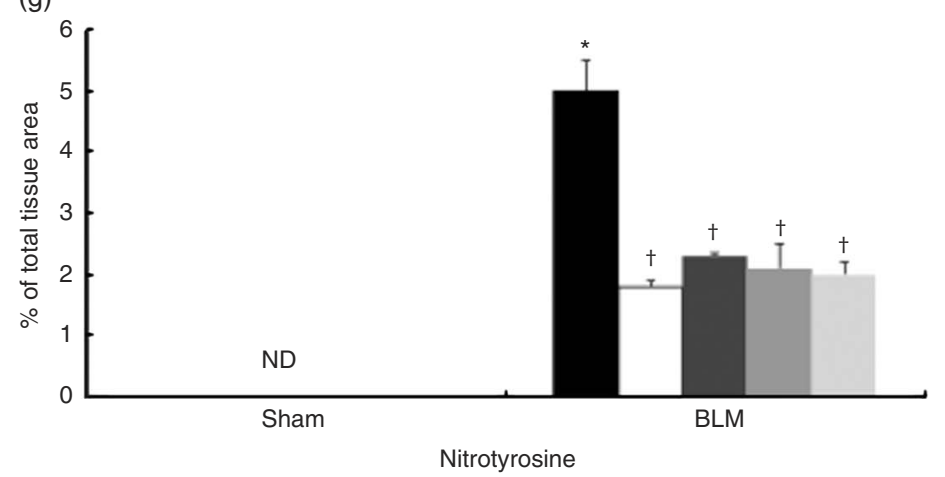

Fig. 3. Effects of resveratrol, mangiferin, dihydroquercetin (DHQ) and quercetin on bleomycin (BLM)-induced nitrotyrosine formation. Immunohistochemical analysis of lung sections obtained from mice treated with BLM revealed positive staining for nitrotyrosine (b). In BLM mice treated with resveratrol, mangiferin, DHQ and quercetin, positive staining for nitrotyrosine was significantly reduced (c-f). No positive staining for nitrotyrosine was observed in lung tissues obtained from shamoperated mice (a). Densitometry analysis $(\mathrm{g})$ of immunohistochemistry photographs ( $n 5$ photos from each sample collected from all mice in each experimental group) was assessed. The assay was carried out using AxioVision on a personal computer. The figure is representative of at least three experiments performed on different experimental days. Data are expressed as percentages of total tissue area and are mean values and standard deviations from $n 10$ mice for each group. * $P<0.05 \mathrm{~V}$. sham; $\uparrow P<0.05$ v. BLM. $\square$, Vehicle; $\square$, resveratrol; , mangiferin; , DHQ; , quercetin.

quercetin attenuated the development of BLM-induced lung injury. NF- $\kappa$ Bp 65 levels in the lung nuclear fractions were significantly increased after BLM injection compared with the sham-treated mice (Fig. 7(a)). Treatment with resveratrol and mangiferin significantly reduced the levels of NF- $\kappa \mathrm{Bp} 65$ (Fig. 5(b)). In contrast, DHQ and quercetin administration was not able to reduce significantly the expression of NF- $\kappa \mathrm{Bp} 65$ (Fig. 7(a)). Moreover, basal expression of $\mathrm{I} \kappa \mathrm{B}-\alpha$ was detected in lung samples from sham-treated animals, whereas I $\kappa \mathrm{B}-\alpha$ levels were substantially reduced in lung tissues obtained from vehicle-treated animals after BLM injection (Fig. 7(b)). Resveratrol and mangiferin significantly prevented BLM-induced I $\kappa \mathrm{B}-\alpha$ degradation (Fig. 7(b)). In contrast, DHQ and quercetin administration was not able to reduce $\mathrm{I} \kappa \mathrm{B}-\alpha$ degradation significantly (Fig. 7(a)).

\section{Discussion}

Lung fibrosis is the common end point of ILD, characterised by chronic inflammation and progressive fibrosis of the pulmonary interstitium $^{(32)}$. Management of IPF is highly debatable and no curative treatment has been developed so far. Corticosteroids, immunosuppressive or cytotoxic agents are the first choice of drugs in common practice. However, benefit of these treatment options is unclear while having potentially serious side-effects ${ }^{(33)}$. During recent years, there has been a growing interest in the therapeutic use of natural compounds to prevent tissue damage induced by oxidative stress and pro-inflammatory mediators ${ }^{(34)}$. Numerous studies have demonstrated the potent antioxidative and anti-inflammatory properties of plant polyphenols present in fruits, and it has been 
(a)

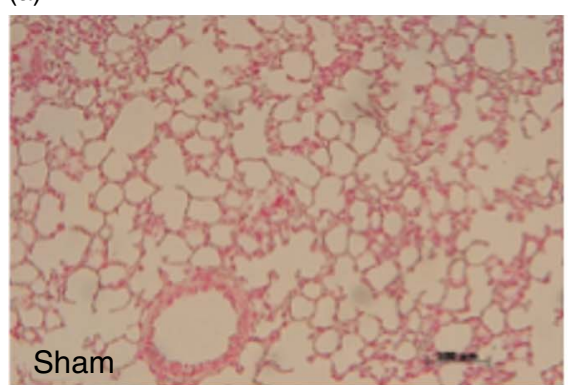

(d)

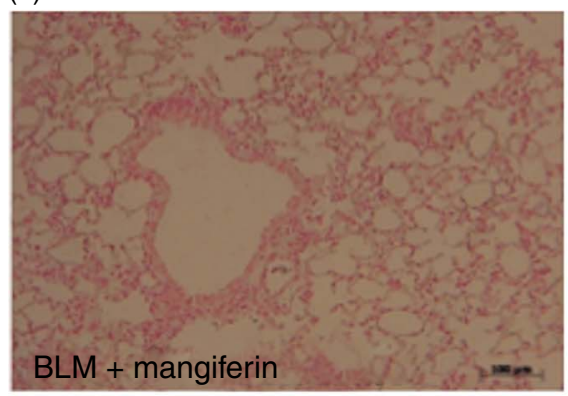

(b)

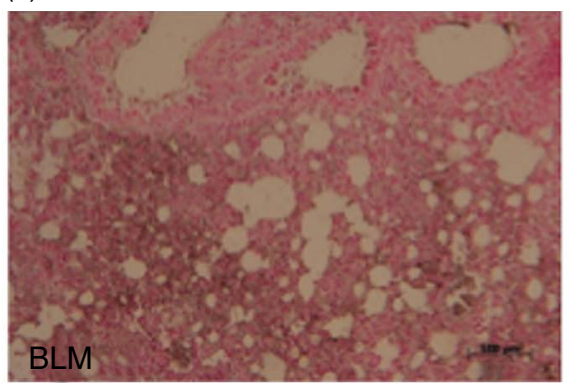

(e)

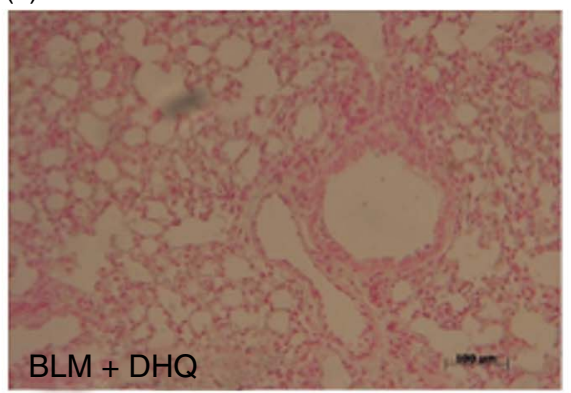

(c)

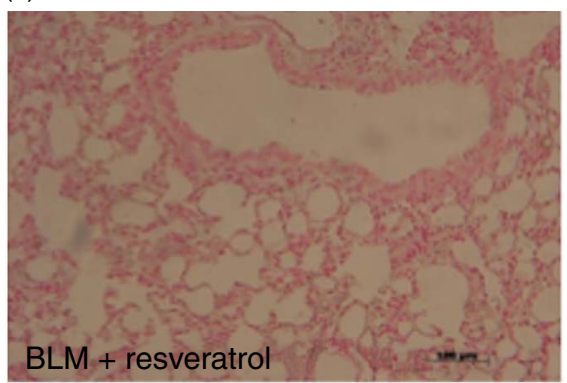

(f)

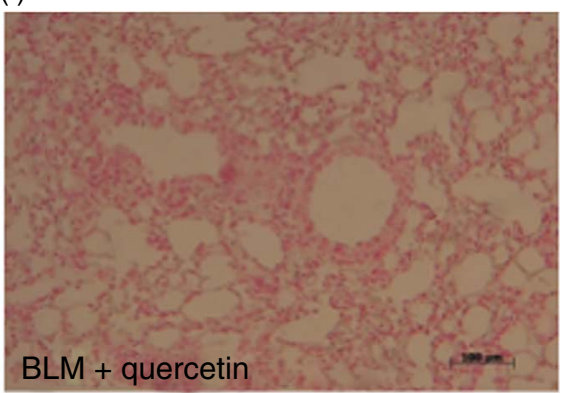

(g)

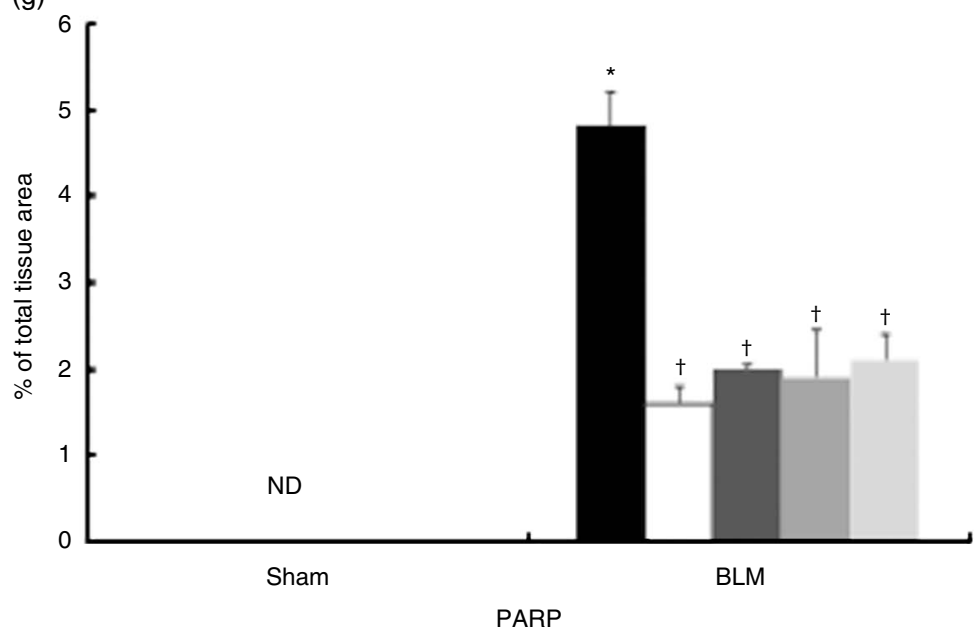

Fig. 4. Effects of resveratrol, mangiferin, dihydroquercetin (DHQ) and quercetin on bleomycin (BLM)-induced poly (ADP-ribose) polymerase (PARP) formation. Immunohistochemical analysis of lung sections obtained from mice treated with BLM revealed a positive staining for PARP (b). In contrast, positive staining for PARP was significantly reduced in the lungs of BLM mice treated with resveratrol, mangiferin, DHQ and quercetin (c-f). No positive staining for PARP was observed in lung tissues obtained from sham-operated mice (a). Densitometry analysis $(\mathrm{g})$ of immunohistochemistry photographs ( $n 5$ photos from each sample collected from all mice in each experimental group) was assessed. The assay was carried out using AxioVision on a personal computer. The figure is representative of at least three experiments performed on different experimental days. Data are expressed as percentages of total tissue area and are mean values and standard deviations from $n 10$ mice for each group. * $P<0.05$ v. sham; $\dagger P<0.05$ v. BLM. $\square$, Vehicle; $\square$, resveratrol; $\square$, mangiferin;, DHQ; , quercetin; ND, not detected.

suggested that these substances can be used as therapeutics for inflammation-related diseases ${ }^{(35,36)}$. Based on this background, in our study, we examined the effects of administration of resveratrol and quercetin as well as a mango leaf extract, rich in mangiferin, and a grape leaf extract, rich in DHQ, in the BLM mouse model of lung fibrosis to assess the therapeutic potential of these polyphenols in the fibrosis setting.

Intra-tracheal instillation of the anti-tumour agent BLM is the most commonly used animal model for pulmonary fibrosis ${ }^{(37)}$. In our study, the injection of BLM elicited an inflammatory response, characterised by the accumulation of fluid in the lungs, an indicator of oedema, accompanied by a marked reduction in the body weight. Treatment of mice with resveratrol, mangiferin, quercetin and DHQ inhibited oedema formation and body weight loss of the mice while improving the histopathological damage of pulmonary tissue induced by BLM. The BLM-induced lung inflammation is orchestrated in part by neutrophils and macrophages. After BLM injection, these inflammatory cells are activated and migrate into the inflammatory foci where they release ROS, proteases and inflammatory cytokines that sustain the injury/repair processes that are considered to contribute to the fibrotic processes induced 
(a)
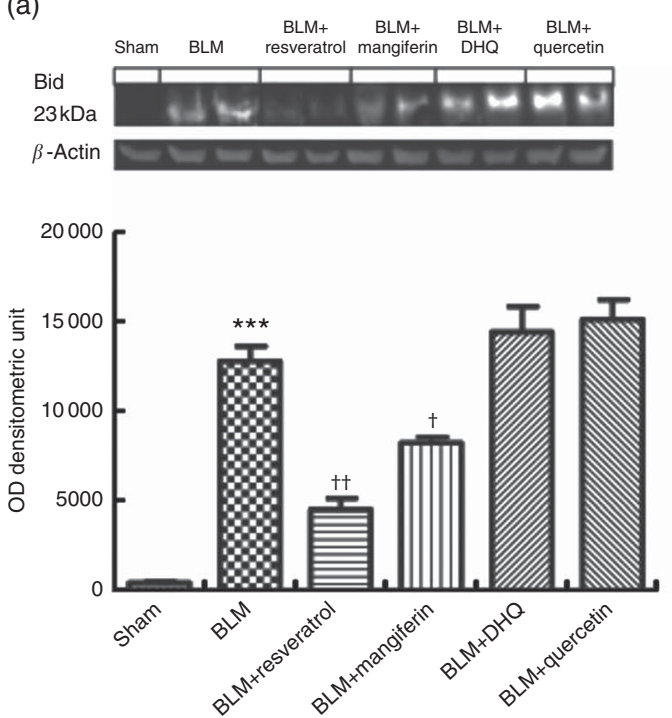

(b)
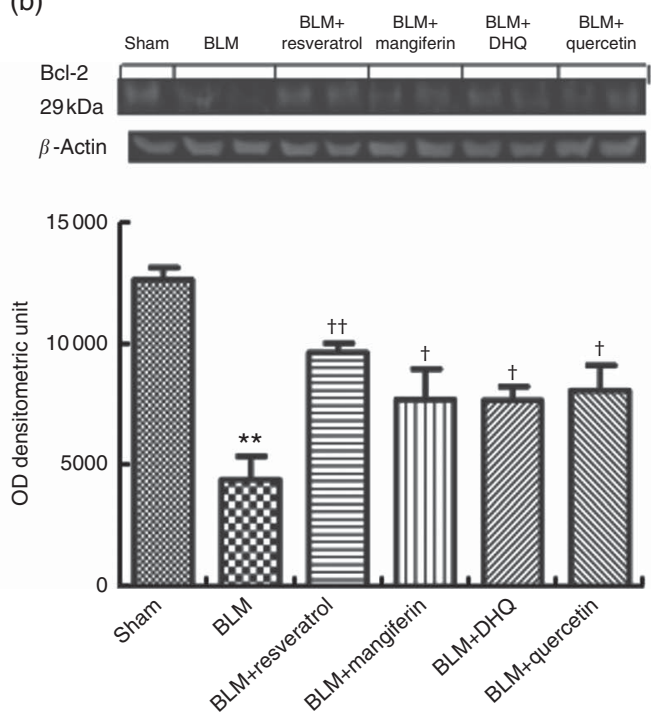

Fig. 5. Effect of resveratrol, mangiferin, dihydroquercetin (DHQ) and quercetin on apoptotic expression levels. A significant increase in Bid levels was observed in lung sections obtained from mice treated with bleomycin (BLM); on the contrary, treatment with resveratrol and mangiferin was able to reduce this protein expression (a). Moreover, a significant decrease in Bcl-2 levels was observed in lung sections obtained from mice treated with BLM; on the contrary, treatment with resveratrol, mangiferin, $\mathrm{DHQ}$ and quercetin was able to increase $\mathrm{Bcl}-2$ expression (b). $\beta$-Actin was used as the internal control. A representative blot of lysates obtained from each group is shown, and densitometry analysis of all animals is reported ( $n 10$ mice from each group). ${ }^{\star *} P<0.01 \mathrm{v}$. sham; ${ }^{\star \star *} P<0.001 \mathrm{v}$. sham; $\dagger P<0.05 \mathrm{v}$. BLM; †† $P<0.01$ v. BLM.

by $\mathrm{BLM}^{(38)}$. MPO activity is used as a quantitative measure of neutrophil inflammatory response; this enzyme activity has been shown to be increased in lung tissues and BAL fluid from patients with $\mathrm{IPF}^{(39)}$. In this study, BLM administration resulted in increased MPO activity and enhanced neutrophil and macrophage trafficking into air spaces that accentuated inflammatory response in BLM-induced mice. On the contrary, treatment with polyphenols ameliorated polymorphonuclear infiltration into the lung tissue, as evidenced by the suppression of MPO activity, as well as reduced the number of inflammatory cells in BAL fluid after $7 \mathrm{~d}$ of BLM instillation. These inhibitory effects of polyphenols on the accumulation of leucocytes into the lung may contribute to a further protection of the lung from free radical damage produced by leucocytes, thereby alleviating tissue damage in this organ. Consistent with these findings, resveratrol - administered either in the early phase or in the late phase of lung fibrosis - as well as a treatment with quercetin, or liposomal quercetin, reduced the total cell count and the number of neutrophils in BAL fluids ${ }^{(21,22,24,25)}$ from BLM-instilled animals. In a similar way, administration of mangiferin suppressed the influx of leucocytes into BAL fluid in a model of allergic asthma as well as in a model of sepsis-induced lung injury ${ }^{(26,27)}$.

There is strong evidence that the cellular redox state and the oxidant/antioxidant balance play important roles in the pathogenesis of $\mathrm{IPF}^{(40)}$. Endogenous NO levels play a key role in physiological regulation of airway functions and its overproduction is implicated in airway disease. NO mediates vasodilation and bronchodilatation; it is synthesised from L-arginine by two constitutive forms of NOS, which are involved in the physiological regulation of airway function ${ }^{(41)}$. The inducible enzyme iNOS is able to generate much larger quantities of NO than the constitutive isoforms, and it is directly involved in host defence against infections ${ }^{(42)}$ as well as in the initiation and maintenance of different experimental and clinical pathologies including lung diseases ${ }^{(43,44)}$. Exogenous NO stimulates in vitro fibroblast proliferation ${ }^{(45)}$, and iNOS up-regulation in lung fibroblasts is associated with the early proliferative response to cytokine stimulation $^{(46)}$. In our study, the Western blot results showed an increase in iNOS expression in the lung tissues after BLM administration; on the contrary, treatment with resveratrol, mangiferin, DHQ and quercetin reduced the enzyme levels in lung tissue. It has been shown that increased production of $\mathrm{NO}$ combined with the production of ROS contributes to the formation of highly reactive products such as peroxynitrite. The formation of nitrotyrosine is a marker for the detection of the generation of peroxynitrite and other stress nitrosative derivatives $^{(37)}$. Our results reveal that exogenous administration of the different polyphenols attenuated the increase of nitrotyrosine formation caused by BLM instillation. We propose that the reduction of nitrosative stress by these polyphenols may be attributed to the inhibition of iNOS expression, and the subsequent formation of NO. Over-production of reactive oxygen and nitrogen intermediates may provoke DNA breakage and can lead to PARP activation. Continuous and excessive PARP activation produces extended chains of ADP-ribose on nuclear proteins and results in a substantial depletion of intra-cellular $\mathrm{NAD}+$ and subsequently ATP, leading to cellular dysfunction and cell death ${ }^{(47)}$. In this study, we demonstrate that treatments with resveratrol, mangiferin, DHQ and quercetin were able to reduce the increase in PARP activation in the lungs from BLM-treated mice. Taken together, our findings report that these polyphenols reduced the expression of markers of nitrosative and oxidative stress, and thus DNA damage in lung 
(a)
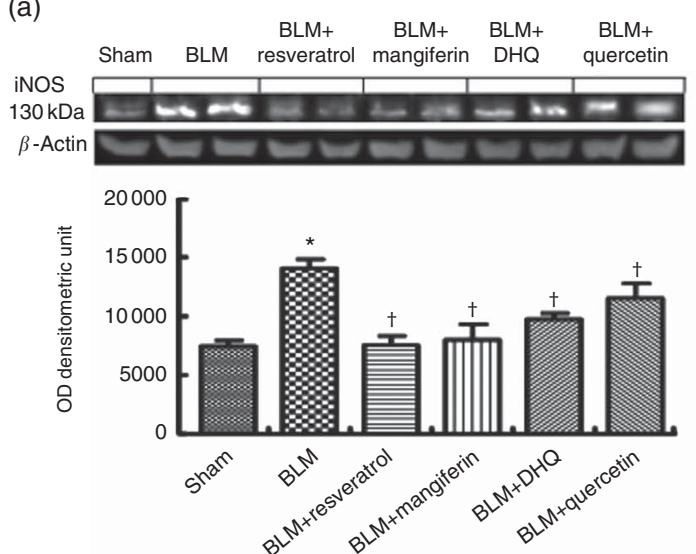

(b)
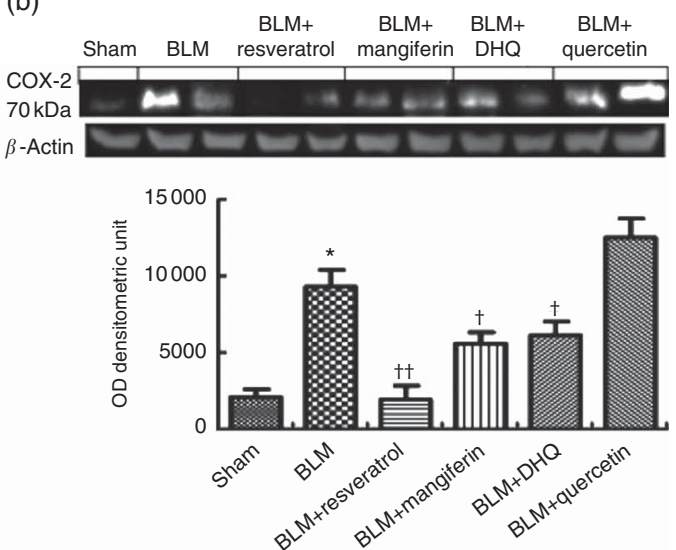
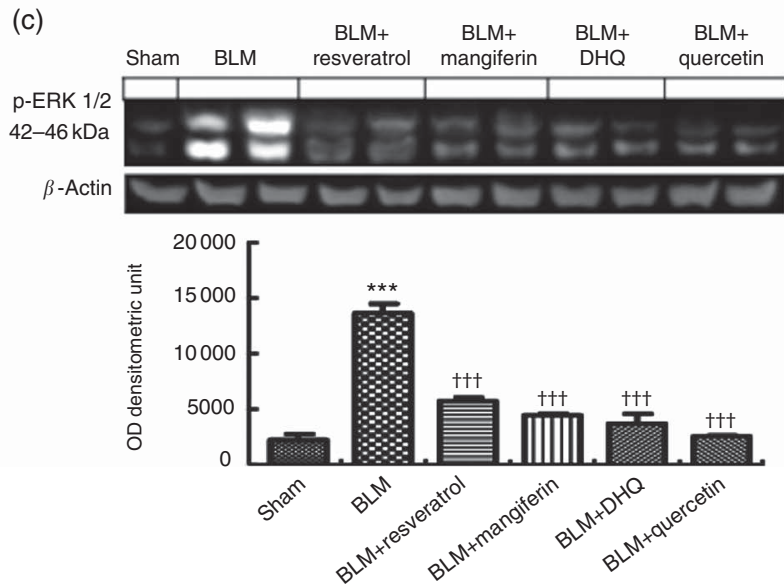

Fig. 6. Effect of resveratrol, mangiferin, dihydroquercetin (DHQ) and quercetin on inducible nitric oxide synthase (iNOS), cyclo-oxygenase-2 (COX-2) and extracellular signal-regulated kinase ( $p E R K$ ) expressions. A significant increase of iNOS, COX-2 and $p E R K$ levels was observed in lung sections obtained from mice treated with bleomycin (BLM) (a-c, respectively). The treatment with resveratrol, mangiferin, DHQ and quercetin significantly reduced iNOS, COX-2 and $p E R K$ levels (a-c, respectively). Data were normalised on the basis of $\beta$-actin levels. A representative blot of lysates obtained from each group is shown, and densitometry analysis of all animals is reported $\left(n 10\right.$ mice from each group). ${ }^{*} P<0.05 \mathrm{v}$. sham; ${ }^{* \star \star} P<0.001 \mathrm{v}$. sham; $\dagger P<0.05 \mathrm{v}$. BLM; $† \dagger P<0.01 \mathrm{v}$. BLM; ††† $P<0.001 \mathrm{v}$. BLM.

tissue. In agreement with our results, previous studies have reported the antioxidant properties of resveratrol and quercetin in experimental models of lung injury, through inhibition of malondialdehyde levels, an index of lipid peroxidation, and increase in the activity of the antioxidant enzymes superoxide dismutase, catalase and glutathione ${ }^{(21,22,24,25)}$.

Recent studies have implicated apoptosis of alveolar epithelial cells as a potential initiating mechanism in the development of lung diseases ${ }^{(48)}$. It has been shown that BLM induces apoptosis of these cells through increased production of ROS $^{(49)}$. Our findings reported a reduction of cellular apoptosis in lungs from BLM-treated mice administered with polyphenols, especially resveratrol and mangiferin, evidenced by the attenuation of the pro-apoptotic protein Bid and the increase of the anti-apoptotic protein Bcl-2. It is also known that ROS can stimulate the synthesis of the pro-inflammatory enzyme COX-2 and PG, which have been shown to be involved in the pathogenesis of lung fibrosis ${ }^{(50)}$. In our study, lung damage was associated with a high expression of COX-2 protein, which was significantly reduced after treatment with polyphenols. In line with these results, previous studies have reported the inhibitory effects of resveratrol and quercetin on COX-2 expression in experimental septic acute lung injury induced by lipopolysaccharides ${ }^{(51)}$. Likewise, mangiferin down-regulated the expression of COX-2 in a murine model of sepsis-induced lung injury $^{(27)}$.

Several researchers have demonstrated that ROS can induce the activation of signalling pathways such as MAP kinases or $N F-\kappa B^{(52)}$. Various experimental evidences have clearly suggested that NF- $\kappa \mathrm{B}$ plays a central role in the regulation of many genes responsible for the generation of mediators or proteins in lung inflammation such as TNF- $\alpha$, IL- $1 \beta$, iNOS and COX-2. By inhibiting the activation of $\mathrm{NF}-\kappa \mathrm{B}$, the production of inflammatory mediators may be reduced as well ${ }^{(53)}$. A number of cellular stimuli that induce ROS production also in parallel can activate MAPK pathways in multiple cell types. When MAPKs, including ERK1/2, p38 and JNK, are activated by inflammatory stimuli, these signal molecules are phosphorylated, resulting in the production of pro-inflammatory mediators such as cytokines, COX-2 and iNOS. ERK is a member of the MAPK family that play a crucial role in signal transduction and regulate cell death and survival ${ }^{(54)}$. To further define the possible 
(a)

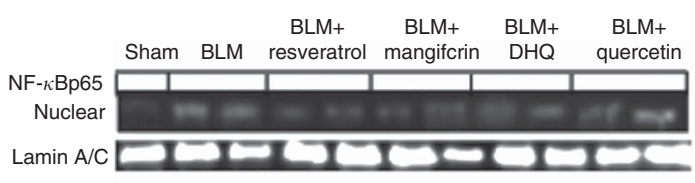

(b)
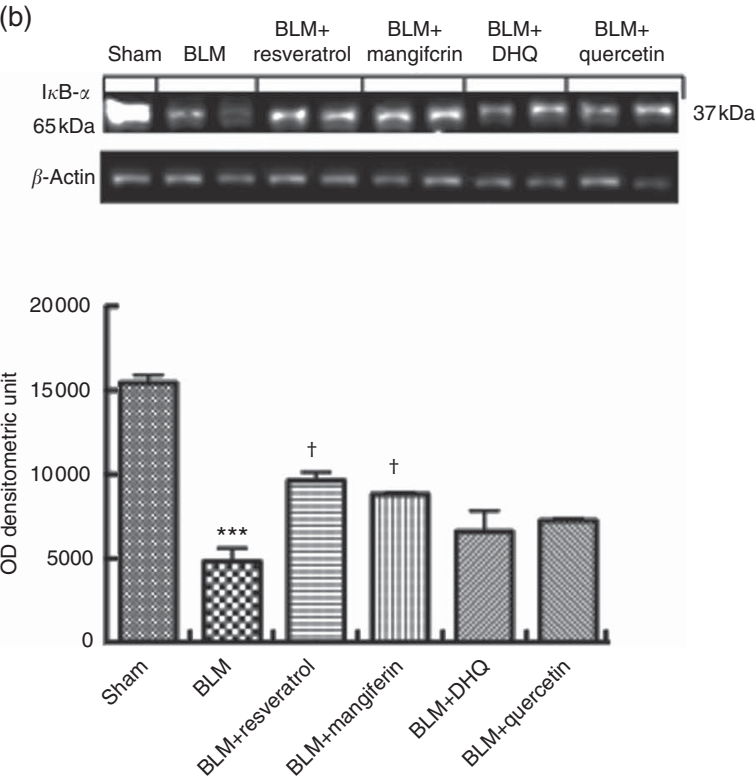

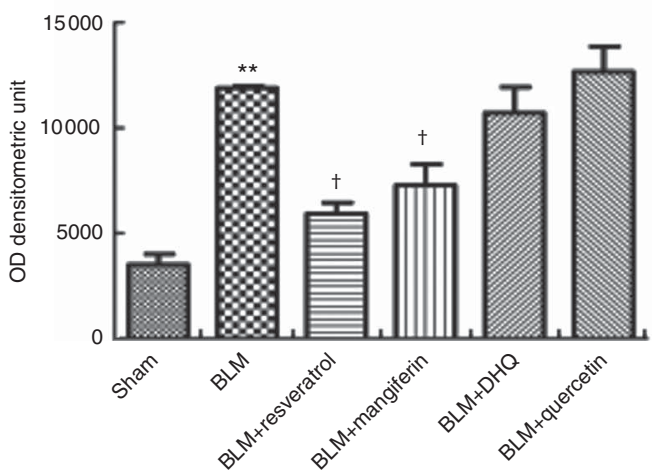

Fig. 7. Effect of resveratrol, mangiferin, dihydroquercetin (DHQ) and quercetin on IKB- $a$ degradation and NF-kBp65 translocation. Using Western blot analysis, $\mathrm{NF}-k B$ B65 levels in the lung nuclear fractions were increased after bleomycin (BLM) injection compared with the sham-treated mice (a). Resveratrol and mangiferin reduced the levels of NF-kBp65 (a). DHQ and quercetin were not able to reduce significantly NF- $k$ Bp65 levels (a). In addition, basal expression of $I k B-a$ was detected in lung samples from sham-treated animals, whereas $1 k B-a$ levels were substantially reduced in lung tissues obtained from vehicle-treated animals after BLM injection (b). Resveratrol and mangiferin prevented BLM-induced $\mathrm{I} \mathrm{KB}-\alpha$ degradation in a significant manner (b). $\beta$-Actin and lamin $\mathrm{A} / \mathrm{C}$ were used as internal control. A representative blot of lysates obtained from each group is shown, and densitometry analysis of all animals is reported $\left(n 10\right.$ mice from each group). ${ }^{* \star} P<0.01 \mathrm{~V}$. sham; ${ }^{* *} P<0.001$ v. sham; $† P<0.05$ v. BLM.

mechanisms of resveratrol, mangiferin, quercetin and DHQ in regulating BLM-induced lung injury, we examined their effects on pERK, NF- $\kappa \mathrm{B}$ and $\mathrm{I} \kappa \mathrm{B}-\alpha$. In keeping with the inhibition of the pro-inflammatory enzymes as mentioned above, resveratrol, mangiferin, DHQ and quercetin prevented ERK activation, and in particular resveratrol and mangiferin reduced $\mathrm{I} \kappa \mathrm{B}-\alpha$ degradation and NF- $\kappa$ Bp 65 nuclear translocation, suggesting that these polyphenols provide early protection in the BLM-injured mice via inhibiting MAPKs as well as NF- $\kappa$ B pathways. In support of this finding, resveratrol has been shown to inhibit transforming growth factor- $\beta$-induced phosphorylation of ERK in human pulmonary fibroblasts, resulting in a reduced cell proliferation $^{(55)}$. In addition, administration of mangiferin attenuated the activation of MAPKs signalling, and as a consequence inhibited the production of COX- 2 and iNOS in a model of sepsis-induced lung injury in mice ${ }^{(27)}$. In that regard, previous studies have also shown that resveratrol suppressed phosphorylation and subsequent degradation of $\mathrm{I} \kappa \mathrm{B}-\alpha$, thereby inhibiting activation of NF- $\kappa \mathrm{B}$ in TPA-stimulated mouse skin ${ }^{(56)}$ and mangiferin-protected rats against chronic bronchitis via regulating NF- $\kappa \mathrm{Bp} 65$ and $\mathrm{I} \kappa \mathrm{B}-\alpha$ expressions ${ }^{(57)}$. In summary, our results provide new insights into the molecular mechanisms by which resveratrol and quercetin exert their beneficial effects on BLM-lung injury and highlight, for the first time, the protective properties of exogenous administration of a mango leaf extract, rich in mangiferin, and a grape leaf extract, rich in DHQ, on experimental pulmonary fibrosis. Our findings indicated that the protective effects of polyphenols on BLM-induced lung injury may be related to the decrease in neutrophil migration and nitrosative and oxidative stress during the course of the inflammatory response. The prevention of ROS accumulation by polyphenols could inhibit apoptosis as well as block MAPK activation and the subsequent production of pro-inflammatory enzymes such as iNOS and COX-2, suggesting that these natural products could be used therapeutically for controlling inflammation-related diseases including IPF.

\section{Acknowledgements}

The authors thank Giovanni Leotta and Antonietta Medici for their excellent technical assistance during this study and Ms Valentina Malvagni for editorial assistance with the manuscript.

This work was supported by a grant from the Italian Health Ministry (PRIN).

This study is a collaboration research between both groups from the University of Seville and the University of Messina and was coordinated by V. M. and S. C., respectively.

There are no conflicts of interest.

\section{References}

1. Sleijfer S (2001) Bleomycin-induced pneumonitis. Chest 120, 617-624.

2. Lazenby AJ, Crouch EC, McDonald JA, et al. (1990) Remodeling of the lung in bleomycin-induced pulmonary fibrosis in the rat. An immunohistochemical study of laminin, type IV collagen, and fibronectin. Am Rev Respir Dis 142, 206-214.

3. Walsh J, Absher M \& Kelley J (1993) Variable expression of platelet-derived growth factor family proteins in acute lung injury. Am J Respir Cell Mol Biol 9, 637-644. 
4. Pron G, Belehradek J Jr, Orlowski S, et al. (1994) Involvement of membrane bleomycin-binding sites in bleomycin cytotoxicity. Biochem Pharmacol 48, 301-310.

5. Goodman MT, Hernandez B, Wilkens LR, et al. (1998) Effects of beta-carotene and alpha-tocopherol on bleomycin-induced chromosomal damage. Cancer Epidemiol Biomarkers Prev 7, 113-117.

6. Hubbard AK, Timblin CR, Shukla A, et al. (2002) Activation of NF-kappaB-dependent gene expression by silica in lungs of luciferase reporter mice. Am J Physiol Lung Cell Mol Physiol 282, L968-L975.

7. Saleh D, Barnes PJ \& Giaid A (1997) Increased production of the potent oxidant peroxynitrite in the lungs of patients with idiopathic pulmonary fibrosis. Am J Respir Crit Care Med 155, 1763-1769.

8. Middleton E Jr, Kandaswami C \& Theoharides TC (2000) The effects of plant flavonoids on mammalian cells: implications for inflammation, heart disease, and cancer. Pharmacol Rev 52, 673-751.

9. Chuang CC \& McIntosh MK (2011) Potential mechanisms by which polyphenol-rich grapes prevent obesity-mediated inflammation and metabolic diseases. Annu Rev Nutr 31, 155-176.

10. Valenzano DR, Terzibasi E, Genade T, et al. (2006) Resveratrol prolongs lifespan and retards the onset of age-related markers in a short-lived vertebrate. Curr Biol 16, 296-300.

11. Elmali N, Baysal O, Harma A, et al. (2007) Effects of resveratrol in inflammatory arthritis. Inflammation 30, 1-6.

12. Vang O (2013) What is new for resveratrol? Is a new set of recommendations necessary? Ann NY Acad Sci 1290, $1-11$.

13. Boots AW, Haenen GR \& Bast A (2008) Health effects of quercetin: from antioxidant to nutraceutical. Eur J Pharmacol $\mathbf{5 8 5}, 325-337$.

14. Wang RR, Gao YD, Ma CH, et al. (2011) Mangiferin, an antiHIV-1 agent targeting protease and effective against resistant strains. Molecules 16, 4264-4277.

15. Li X, Cui X, Sun X, et al. (2010) Mangiferin prevents diabetic nephropathy progression in streptozotocin-induced diabetic rats. Phytother Res 24, 893-899.

16. Slimestad R, Fossen $\mathrm{T}$ \& Vagen IM (2007) Onions: a source of unique dietary flavonoids. J Agric Food Chem 55, 10067-10080.

17. Wallace SN, Carrier DJ \& Clausen EC (2005) Batch solvent extraction of flavanolignans from milk thistle (Silybum marianum L. Gaertner). Phytochem Anal 16, 7-16.

18. Rohdewald P (2002) A review of the French maritime pine bark extract (Pycnogenol), a herbal medication with a diverse clinical pharmacology. Int J Clin Pharmacol Ther $\mathbf{4 0}$, $158-168$.

19. Murach EI, Baranov IA, Erlykina EI, et al. (2014) Adaptogenic effects of dihydroquercetin-chitosan composition during modeling of acute hypoxia. Bull Exp Biol Med 156, 306-309.

20. Weidmann AE (2012) Dihydroquercetin: more than just an impurity? Eur J Pharmacol 684, 19-26.

21. Sener G, Topaloglu N, Sehirli AO, et al. (2007) Resveratrol alleviates bleomycin-induced lung injury in rats. Pulm Pharmacol Ther 20, 642-649.

22. Akgedik R, Akgedik S, Karamanli H, et al. (2012) Effect of resveratrol on treatment of bleomycin-induced pulmonary fibrosis in rats. Inflammation 35, 1732-1741.

23. Yuan ZP, Chen LJ, Fan LY, et al. (2006) Liposomal quercetin efficiently suppresses growth of solid tumors in murine models. Clin Cancer Res 12, 3193-3199.

24. Baowen Q, Yulin Z, Xin W, et al. (2010) A further investigation concerning correlation between anti-fibrotic effect of liposomal quercetin and inflammatory cytokines in pulmonary fibrosis. Eur J Pharmacol 642, 134-139.

25. Verma R, Kushwah L, Gohel D, et al. (2013) Evaluating the ameliorative potential of quercetin against the bleomycininduced pulmonary fibrosis in Wistar rats. Pulm Med 2013, 921724 .

26. Guo HW, Yun CX, Hou GH, et al. (2014) Mangiferin attenuates TH1/TH2 cytokine imbalance in an ovalbumin-induced asthmatic mouse model. PLOS ONE 9, e100394.

27. Gong X, Zhang L, Jiang R, et al. (2013) Anti-inflammatory effects of mangiferin on sepsis-induced lung injury in mice via up-regulation of heme oxygenase-1. J Nutr Biochem 24, $1173-1181$

28. Ma L, Li Y, Zhao Y, et al. (2013) 3,5,4'-tri-O-acetylresveratrol ameliorates seawater exposure-induced lung injury by upregulating connexin 43 expression in lung. Mediators Inflamm 2013, 182132

29. Galindo P, Gonzalez-Manzano S, Zarzuelo MJ, et al. (2012) Different cardiovascular protective effects of quercetin administered orally or intraperitoneally in spontaneously hypertensive rats. Food Funct 3, 643-650.

30. Ashcroft T, Simpson JM \& Timbrell V (1988) Simple method of estimating severity of pulmonary fibrosis on a numerical scale. J Clin Pathol 41, 467-470.

31. Mullane KM, Kraemer R \& Smith B (1985) Myeloperoxidase activity as a quantitative assessment of neutrophil infiltration into ischemic myocardium. J Pharmacol Methods 14, $157-167$.

32. Green FH (2002) Overview of pulmonary fibrosis. Chest 122, 334S-339S

33. Antoniou KM, Margaritopoulos GA \& Siafakas NM (2013) Pharmacological treatment of idiopathic pulmonary fibrosis: from the past to the future. Eur Respir Rev 22, 281-291.

34. Skrovankova S, Misurcova L \& Machu L (2012) Antioxidant activity and protecting health effects of common medicinal plants. Adv Food Nutr Res 67, 75-139.

35. Gonzalez-Munoz A, Quesille-Villalobos AM, Fuentealba C, et al. (2013) Potential of Chilean native corn (Zea mays L.) accessions as natural sources of phenolic antioxidants and in vitro bioactivity for hyperglycemia and hypertension management. I Agric Food Chem 61, 10995-11007.

36. Di Carlo G, Mascolo N, Izzo AA, et al. (1999) Flavonoids: old and new aspects of a class of natural therapeutic drugs. Life Sci 65, 337-353.

37. Di Paola R, Talero E, Galuppo M, et al. (2011) Adrenomedullin in inflammatory process associated with experimental pulmonary fibrosis. Respir Res 12, 41.

38. Galuppo M, Di Paola R, Mazzon E, et al. (2010) GW0742, a high affinity PPAR-beta/delta agonist reduces lung inflammation induced by bleomycin instillation in mice. Int $J$ Immunopathol Pharmacol 23, 1033-1046.

39. Hallgren R, Bjermer L, Lundgren R, et al. (1989) The eosinophil component of the alveolitis in idiopathic pulmonary fibrosis. Signs of eosinophil activation in the lung are related to impaired lung function. Am Rev Respir Dis 139, 373-377.

40. Mastruzzo C, Crimi N \& Vancheri C (2002) Role of oxidative stress in pulmonary fibrosis. Monaldi Arch Chest Dis 57, $173-176$.

41. Moncada S, Palmer RM \& Higgs EA (1989) Biosynthesis of nitric oxide from L-arginine. A pathway for the regulation of cell function and communication. Biochem Pharmacol 38, 1709-1715.

42. McCall TB, Boughton-Smith NK, Palmer RM, et al. (1989) Synthesis of nitric oxide from L-arginine by neutrophils. Release and interaction with superoxide anion. Biochem $J$ 261, 293-296. 
43. Cuzzocrea S, Zingarelli B, Hake $\mathrm{P}$, et al. (1998) Antiinflammatory effects of mercaptoethylguanidine, a combined inhibitor of nitric oxide synthase and peroxynitrite scavenger, in carrageenan-induced models of inflammation. Free Radic Biol Med 24, 450-459.

44. Wei XQ, Charles IG, Smith A, et al. (1995) Altered immune responses in mice lacking inducible nitric oxide synthase. Nature 375, 408-411.

45. Gansauge S, Gansauge F, Nussler AK, et al. (1997) Exogenous, but not endogenous, nitric oxide increases proliferation rates in senescent human fibroblasts. FEBS Lett $\mathbf{4 1 0}$ $160-164$.

46. Romanska HM, Polak JM, Coleman RA, et al. (2002) iNOS gene upregulation is associated with the early proliferative response of human lung fibroblasts to cytokine stimulation. J Pathol 197, 372-379.

47. Chiarugi A (2002) Poly(ADP-ribose) polymerase: killer or conspirator? The 'suicide hypothesis' revisited. Trends Pharmacol Sci 23, 122-129.

48. Jin HL \& Dong JC (2011) Pathogenesis of idiopathic pulmonary fibrosis: from initial apoptosis of epithelial cells to lung remodeling? Chin Med J 124, 4330-4338.

49. Wallach-Dayan SB, Izbicki G, Cohen PY, et al. (2006) Bleomycin initiates apoptosis of lung epithelial cells by ROS but not by Fas/FasL pathway. Am J Physiol Lung Cell Mol Physiol 290, L790-L796.

50. Park GY \& Christman JW (2006) Involvement of cyclooxygenase-2 and prostaglandins in the molecular pathogenesis of inflammatory lung diseases. Am J Physiol Lung Cell Mol Physiol 290, L797-L805.

51. Zhang HX, Duan GL, Wang CN, et al. (2014) Protective effect of resveratrol against endotoxemia-induced lung injury involves the reduction of oxidative/nitrative stress. Pulm Pharmacol Ther 27, 150-155.

52. McCubrey JA, Lahair MM \& Franklin RA (2006) Reactive oxygen species-induced activation of the MAP kinase signaling pathways. Antioxid Redox Signal 8, 1775-1789.

53. Impellizzeri D, Bruschetta G, Di Paola R, et al. (2014) The antiinflammatory and antioxidant effects of bergamot juice extract (BJe) in an experimental model of inflammatory bowel disease. Clin Nutr (epublication ahead of print version 27 November 2014).

54. Torres M \& Forman HJ (2003) Redox signaling and the MAP kinase pathways. Biofactors 17, 287-296.

55. Fagone E, Conte E, Gili E, et al. (2011) Resveratrol inhibits transforming growth factor-beta-induced proliferation and differentiation of ex vivo human lung fibroblasts into myofibroblasts through ERK/Akt inhibition and PTEN restoration. Exp Lung Res 37, 162-174.

56. Kundu JK, Shin YK, Kim SH, et al. (2006) Resveratrol inhibits phorbol ester-induced expression of COX-2 and activation of NF-kappaB in mouse skin by blocking IkappaB kinase activity. Carcinogenesis 27, 1465-1474.

57. Wei ZQ, Yan L, Deng JG, et al. (2014) Mangiferin protects rats against chronic bronchitis via regulating NF-kappaB (P65) and IkappaBalpha expression in mononuclear cells. Yao Xue Xue Bao 49, 596-601. 